\title{
FEASIBILITY OF WEARABLE-BASED COLLECTIVE SENSING TO DETECT ENVIRONMENTAL BARRIERS FOR FACILITATING THE ELDERLY'S MOBILITY
}

\author{
G. $\mathrm{Lee}^{1}$, B. $C h o i^{2}$, C.R. $A h n^{3}$, and S. Lee ${ }^{1^{*}}$ \\ ${ }^{1}$ Dept. of Civil and Environmental Engineering, Univ. of Michigan, 2350 Hayward St., Ann Arbor, United States \\ ${ }^{2}$ Dept. of Architectural Engineering, Ajou University, 206, World cup-ro, Suwon-si, Gyeonggi-do, South Korea \\ ${ }^{3}$ Dept. of Construction Science, Texas A\&M Univ., 3137 TAMU, College Station, United States \\ * Corresponding author
}

\begin{abstract}
In our aging society, the elderly's mobility has become critical for our collective prosperity. However, the elderly's mobility is limited in the current built environment due to various types of environmental barriers. Manual surveys have been conducted to detect such environmental barriers, but they are discontinuous, invasive to the elderly's daily lives, and labour-intensive. As such, these methods are not ideal for wider adoption. To continuously, less-invasively, and less-laboriously detect the environmental barriers and advance the elderly's mobility, this study proposes a wearable-based collective sensing approach. This approach measures collective stress, the stress commonly sensed from multiple people on a location, as an indicator of environmental barriers based on people's physiological and location data collected by wearable sensors. To test the feasibility of the proposed approach, a "collective stress metric" is suggested. Then, the values of the collective stress metric on locations of the test site were calculated based on the physiological and location data collected from 10 elderly subjects' daily trips for 2 weeks. Then, every location on the test site was categorized into locations "with environmental barriers" and "without environmental barriers" through site survey. Based on the collected data and results of site survey, the collective stress was statistically compared between locations with environmental barriers and without barriers. The result showed that the collective stress was statistically higher on locations with environmental barriers than without barriers. The results demonstrated that the collective stress has indication of environmental barriers, therefore, the proposed approach is feasible to detect the elderly's environmental barriers.
\end{abstract}

\section{Introduction}

As the rate of the elderly population (i.e., people over the age of 65) is rapidly increasing, elderly individuals' economic and social engagement is critical for our societies' collective prosperity (Walker, 2002). Specifically, mobility, which is the capability to independently access desired places (Metz, 2000), is one fundamental factor of the elderly's economic and social engagement. For example, elderly individuals with impaired mobility tend to engage less frequently in economic and social activities such as shopping, visiting close people and communities (Mollenkopf et al., 1997).

In spite of such importance, the elderly's mobility remains limited (Centers for Disease Control and Prevention, 2018; Guralnik et al., 1993; Manton, 1988). One main reason for the elderly's mobility limitation is their stressful interactions with the current built environment. Although the elderly's physical and cognitive capabilities are impaired by their aging process (Lawton and Nahemow, 1973), the current built environment has not recalibrated for the elderly's impaired capabilities. In the non-recalibrated built environment, there are various types of environmental barriers. The environmental barrier is defined as an element of environment that poses excessive demands (e.g., physical, or cognitive demands) to its individual users (Iwaksson and Isacsson, 1996), thereby limiting or preventing them from fully participating in social, occupational, and recreational activities (Preedy and Watson, 2010). For example, designs for "average person" (e.g., stairs without ramps) (King and Guralnik, 2010; Rantakokko et al., 2009; Rockwood et al., 2004) and environmental defects (e.g., cracked sidewalks and broken stairs) (Bond, 1999) limit the elderly's mobility as the environmental barriers by inducing stress (i.e., a state of mental tension resulting from the excessive demands placed on an individual (Ursin and Eriksen, 2004)) in their daily trips. As a result, several indices of mobility (e.g., trip frequency and trip distance) represent that people's mobility rapidly decreases as they are getting old (Mattson, 2011). Also, the nationwide personal transportation survey noted that the elderly's mobility limitation is substantial because of their stressful interactions with the environmental barriers (Federal Highway Administration, 1995).

To detect such environmental barriers for the elderly's mobility, previous attempts have depended primarily on manual surveys. For example, Lockett et al. (2005) and Rosenberg et al. (2012) identified many different types of environmental barriers that elderly pedestrians have stressful interaction with through focus group interviews. World Health Organization (2007) conducted large-scale interviews with 158 focus group from 33 cities around the world and revealed that insufficient green spaces and places for rest, or fast change in crossing lights can cause stress to the elderly in their trips as an environmental barrier. Although these survey-based attempts 
successfully extended the understanding of the elderly's environmental barriers by identifying various types, these attempts have several limitations that prevent wide practical application. First, since the surveys are typically conducted in a one-time and discontinuous manner, these attempts might miss a substantial number of environmental barriers. For example, various types of "time-dependent" environmental barriers (i.e., environmental barriers that induce pedestrian's stress only in a certain time duration, such as a snowy sidewalk, rain puddles, or risky interaction with automobiles) might not be detected by the discontinuous surveys. Also, the surveys can interfere with elderly individuals' daily lives because the surveys require their active participation (e.g., responses to questionnaires or accompanying to site inspections). Lastly, the survey should be conducted by trained surveyors, so it can be labor-intensive to cover extensive area for a long period. To overcome such limitations, there is an increased need for a continuous, less-disruptive, and less-laborious method to detect the elderly's environmental barriers.

The recent advancements in wearable biosensing technologies have shown a potential for better understanding the peopleenvironment interactions by continuously monitoring people's physiological signals while they interact with their environment. Specifically, recent wearable biosensors that measure people's electrodermal activity (EDA) and photoplethysmogram (PPG) can be used for measuring people' stress in their daily lives. Several physiological activities such as heart activity and sweat production are innervated by people's stress because the arousal of sympathetic nervous system induced by people's stress influences the physiological activities (Cacioppo et al., 2007). With this potential, previous research has applied wearable biosensors to predict people's stress caused by several types of stress stimuli such as emotions (Chang et al., 2013), mental fatigue (Wijsman et al., 2011), and cognitive workload (Setz et al., 2010)) that they can have in their daily lives (e.g., office work, watching movies or driving). In this sense, the wearable biosensors might also be able to detect environmental barriers by continuously measuring people's stress occurring when they interact with the environmental barriers as a stress stimulus.

However, there is a significant knowledge gap to achieve such potential of the wearable biosensor for detection of environmental barriers. Since the physiological data can indicate the level of stress, not its cause, it is hard to distinguish stress caused by the interaction with environmental barriers from ones caused by the other diverse stress stimuli (e.g., emotion, mental fatigue, and cognitive workload). The previous research into the prediction of stress caused by specific stimuli was conducted under controlled experimental settings, which prevent stimuli other than targeted one. In contrast to the controlled experimental settings, it is impossible to control stress stimuli in people's daily lives, so that diverse stimuli might affect people's physiological data. Therefore, a new method that identifies stress caused by environmental barriers from ones by other stimuli is required to detect environmental barriers using wearable biosensors.
To fill this gap, the authors propose the collective sensing (Yang et al., 2017). The collective sensing synthesizes a group of people's signals of interest with location data (e.g., global positioning system (GPS) data) simultaneously to identify locations on which abnormal patterns in the signal occur across the multiple people (Kim et al., 2016; Yang et al., 2017). While a person's stress can be caused by various stimuli, collective stress, which is defined in this study as stress commonly sensed from multiple people on a specific location, can be indicative of a locational stress-inducing factor on the location such as environmental barriers. In this sense, by measuring collective stress on each location using the collective sensing, it is possible to identify locations of environmental barriers. This study aims to test the feasibility of the wearable-based collective sensing to detect the elderly's environmental barriers. Specifically, this study first measures collective stress on each location using wearable sensing, signal processing, and geographical information system (GIS) techniques. Then, the feasibility of the proposed approach is examined by comparing the measured collective stress between locations with environmental barriers and without environmental barriers.

\section{Collective Stress Measurement}

Figure 1 describes the overall process of collective stress measurement based on the proposed wearable-based collective sensing approach. First, elderly individuals' physiological and location data are collected from their daily trips using a set of wearable-type sensors ( $\mathrm{a}$ in Figure 1). Then, individual stress is measured by signal processing techniques such as filtering, decomposition, and segmentation (b in Figure 1). By aggregating multiple elderly people's individual stress on their collected location, collective stress on each location is measured (c in Figure 1).

\section{Figure 1 Collective Stress Measurement Process}

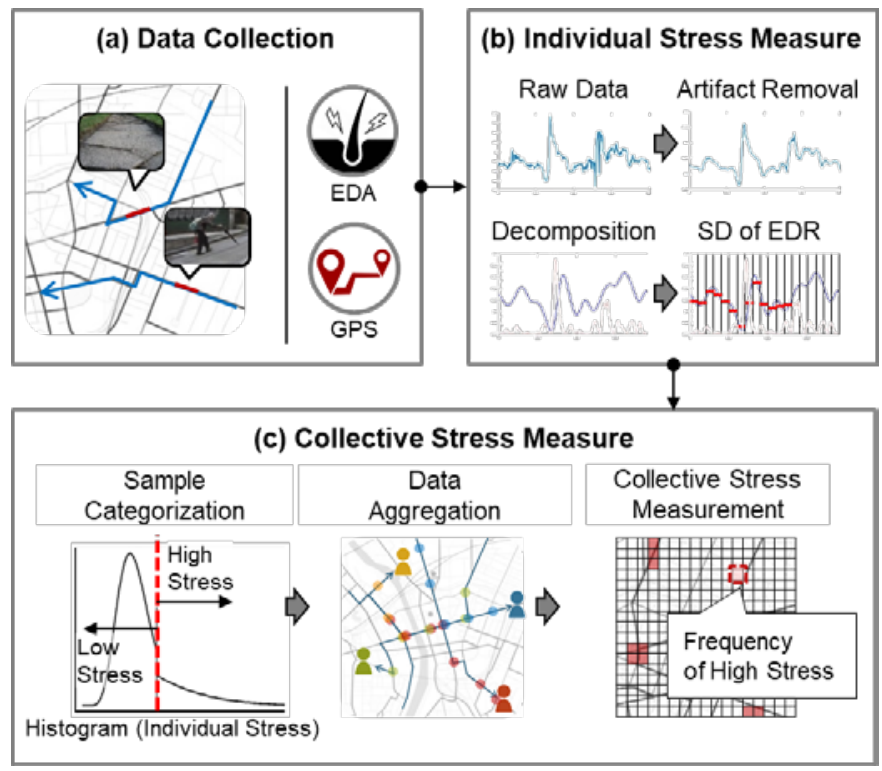




\subsection{Data Collection}

This study uses EDA as physiological data to measure individuals' stress. EDA is changes in electrical properties on the human skin surface induced by eccrine sweat gland activity (Boucsein, 2012). Since the eccrine sweat gland activity is innervated by the arousal of the sympathetic nervous system, which can be induced by stress, EDA can be used to measure people's stress level (Boucsein, 2012). EDA has been more widely used to measure stress than other physiological data such as PPG and electrocardiography (ECG). While EDA is exclusively influenced by the sympathetic nervous system that is related to stress, other physiological data are also innervated by the parasympathetic nervous system that is related to relax. As such, EDA can more reliably measure human stress (Setz et al., 2010). To track elderly individuals' location, GPS data is simultaneously collected together with EDA.

\subsection{Individual Stress Measure}

As a first step of measuring individual stress from EDA, filtering methods are applied to alleviate noises in EDA. EDA collected by wearable-type sensors from daily trips that are uncontrolled and ambulatory contexts may contain diverse noises (i.e., changes in a signal that do not originate from the signal source of interest (Boucsein, 2012)) because of the recording environment and people's physical activities (Heikenfeld et al., 2018). Suppressing such noises is critical to accurately measure stress because the noises can be misinterpreted as reactivity to stress, thereby distorting stress metrics (Sweeney et al., 2012). Two types of filters are applied to alleviate noises in EDA: high-pass filter and moving average filter. A high-pass filter with the cut of the frequency of 0.05 $\mathrm{Hz}$ can mitigate low-frequency noises resulting from variation in temperature, humidity, and impedance of the EDA sensor's electrodes (Jebelli et al., 2018). The moving average filter is used to mitigate high frequency noises in EDA (e.g., noises caused by movement and electrical interference) (Bornoiu and Grigore, 2013; Drachen et al., 2010).

To extract a metric that represents individuals' stress levels from the denoised EDA, the EDA is first decomposed into the electrodermal level (EDL) and electrodermal response (EDR) using the convex optimization method (Greco et al., 2016). While EDL, the tonic component of EDA, reflects the baseline drift of EDA, EDR, the phasic component of EDA, immediately reflects the occurrence of acute stress caused by specific stimuli (Boucsein, 2012). Therefore, the stress metric is extracted based on EDR to immediately capture stress caused by interaction with environmental barriers. Then, the EDR is segmented into samples with 10 seconds length window. The stress metric is extracted from the 10 seconds window EDR samples to fully capture the EDR's reactivity to stress occurrence, which generally has a 10 -second time span (Singh et al., 2014). Standard deviation (SD) of EDR in the 10 seconds window is used as the individual stress metric. SD of EDR has been widely used to measure human stress level because the SD of EDR well represents the intensity of EDR's specific stress reactivity, thus having a significantly positive correlation with the level of stress (Brighetti et al., 2014; Choi et al., 2012; Pappens et al., 2011). As a result, samples that have the value of SD of EDR indicating individual stress levels (the authors call as stress samples from now) are generated from each individual's EDA data.

\subsection{Collective Stress Measure}

To measure collective stress on each location, multiple elderly individuals' stress samples are first aggregated on their collected locations using GIS. Since the raw GPS data is recorded using world geodetic system 1984 (Decker, 1986), it should be projected to conduct the geographical analysis. This study uses NAD 1983 State Plan Michigan South (Michigan Department of Natural Resources, 2005) as a projected coordinate system. This projected coordinate system induces the least distance distortion by projection for the south area in Michigan state. The authors apply a custom grid system in which each grid-cell is $10 \mathrm{~m}$ by $10 \mathrm{~m}$ considering the average walking distance (i.e., 8.3 to $19.4 \mathrm{~m}$ ) of the elderly people for 10 seconds (Bendall et al., 1989), which is the length of a stress sample. Each stress sample is allocated on the grid-cell on which there is the GPS location recorded at the start moment of the stress sample.

As the collective stress metric, this study suggests the frequency of high-stress samples. The frequency of each location indicates how frequently people feel the high level of stress while passing over the location, which is calculated by Equation (1).

$\begin{array}{cc}\text { Frequency of } & \begin{array}{c}\text { Number of } \\ \text { High Stress Samples } \\ \text { on the Grid-cell }\end{array} \\ \text { On a Grid-cell } & =\begin{array}{c}\text { Total Number of } \\ \text { Stress Samples } \\ \text { on the Grid-cell }\end{array}\end{array}$

Since the elderly have a higher chance to become stressed in locations with environmental barriers than locations without barriers, the frequency on each grid-cell can be indicative of the existence of environmental barriers on the grid-cell. For calculating the frequency, it is required to categorize stress samples into "high-stress" and "low-stress." The authors apply the mixture model-based threshold estimation (Behrens et al., 2004; Carreau and Bengio, 2009; Frigessi et al., 2002; MacDonald et al., 2011). This method determines a threshold that separates data into samples with normal values and samples with abnormally high values, which indicate abnormal cases related to specific stimuli. Since the acquired individuals' stress samples were typically observed to be divided into two parts (i.e., the samples with normal values and ones with abnormally high values), the mixture model-based threshold estimation can effectively categorize the stress samples. This method determines the threshold based on the assumption that the samples follow a mixture model containing two separate distributions. Specifically, the former samples with normal values follow a bulk distribution located on the low level and the latter samples with abnormally high values follow the general pareto distribution on the high level. With this 
assumption, the threshold is one of the parameters determining the mixture model, so the threshold can be automatically defined by maximizing the probability that the samples origin from the mixture model. This study uses the kernel density estimator (KDE) to describe the bulk distribution on low level (MacDonald et al., 2011). The KDE is a kernel-based nonparametric way to estimate probabilistic density function from given data (Silverman, 2018). The KDE is more appropriate to explain the stress samples than other parametric distributions such as normal, gamma, and Weibull distributions because the data does not follow such distributions and there is no theoretical background that such parametric distributions well explain the individual stress.

\section{Feasibility Test}

To test the feasibility of the wearable -based collective sensing to detect the elderly's environmental barriers, this study examined whether the collective stress metric (i.e., frequency of high-stress on a grid cell) derived by the collective sensing has indication of environmental barriers or not. Then, physiological and location data were collected from multiple elderly subjects' daily trips using wearable sensors. Also, environmental barriers in the test site were identified through a site survey to categorize grid-cells into with environmental barriers and without. Based on the collected data, values of the collective stress metric on grid-cells in the test site were calculated. Lastly, the feasibility of the proposed method was tested by statistically comparing the collective stress metrics between grid-cells with environmental barriers and without barriers.

\subsection{Field Data Collection}

The test site was a senior apartment (i.e., Clark East Tower senior apartment located in Ypsilanti Township, Michigan, US) and its surrounding areas. The author selected the senior apartment for the data collection for gathering enough data to apply collective sensing within period of data collection. Since residents living there share lots of routes and destinations in their daily trips, it was expected that sufficient data from multiple elderly people would be acquired on the common routes and destination areas within relatively short time. The data collection protocol was approved by the University of Michigan Institutional Review Board.

First, EDA and GPS data were collected from 10 elderly subjects' daily trips. Before the data collection, all the subjects were informed about the anonymity of data collection and subjects' right through the informed consent form. Then, the authors collected the subjects' demographic information (Table 1).

Table 1 Summary of Subjects' Demographic Information

\begin{tabular}{ccccc}
\hline Statistics & Age & Height & Weight & Gender \\
\hline Mean & 67.4 & 164.7 & 86.7 & Male 2 \\
(SD) & $(1.5)$ & $(3.7)$ & $(15.6)$ & Female 8 \\
\hline
\end{tabular}

All the subjects reported no serious problem with activities in their daily trips such as walking and driving. The data was collected for 2 weeks for a subject. The two off-the-shelf wearable sensors (i.e., one wristband-type biosensor for EDA data and one belt-clip-type GPS sensor) were used for this data collection. A 30 minutes training session was provided to help the subjects understand the use of the wearable sensors before collecting the data. During the data collection, the authors visited the subjects twice a week to maintain the sensors and download data from the sensors' storage.

After the collection of physiological and location data, the site survey was conducted to identify environmental barriers in the site. Since values of collective stress from multiple subjects are compared to test feasibility, areas on which only one subject's data was located were excluded in this study. The authors first created a list of the potential environmental barriers of elderly people (e.g., lack of curb ramps, narrow or cracked sidewalks) based on previous research (Lockett et al., 2005; Rosenberg et al., 2012). The site survey was conducted based on the list. As a result, the authors identified six environmental barriers on the test site (Figure 2). Barrier 1 was the curb without ramp, which was located on the residence parking lots' main route. Barriers 2 and 3 were vertical displacement over $1 \mathrm{~cm}$ on sidewalks. A side-sloped sidewalk and obstructed sidewalk were detected as Barriers 4 and 5. Lastly, barrier 6 was the unpaved sidewalk locating between the apartment and the closest commercial building.

\section{Figure 2 Identified Environmental Barriers on the Test Site}

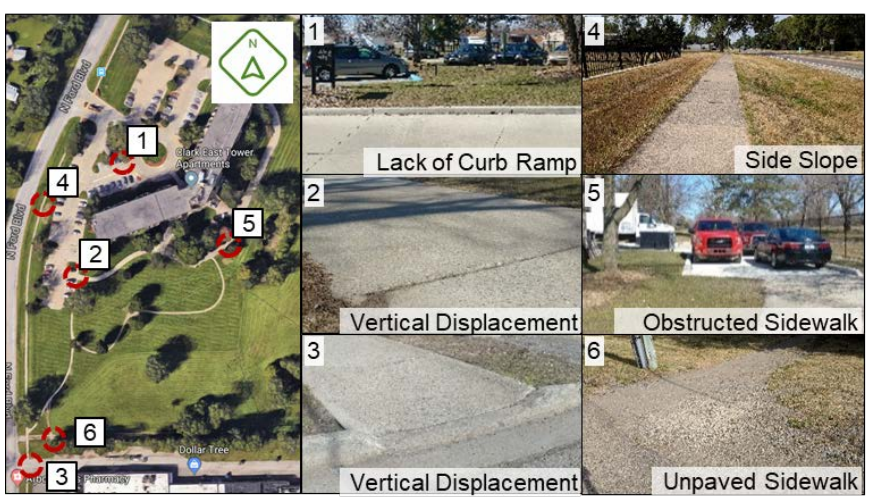

\subsection{Statistical Comparison of Collective Stress}

Based on the result of site survey, every grid-cell was divided into "with environmental barrier" and "without environmental barrier." Among total 231 grid-cells in the test site (except for the grid-cells with less than two subjects' data), six grid-cells are classified as "with environmental barriers" and other 225 grid-cell are classified as "without environmental barriers." Also, collective stress on every grid-cell in the test site were calculated using multiple elderly subjects' data collected from their daily trips. Then, a statistical test is conducted to examine difference in the collective stress between grid-cells with environmental barriers and without environmental barriers. Since the data has significant skewness (i.e., number of gridcells with environmental barrier is far less than that of gridcells without barriers) and the normal distribution could not be 
assumed for the grid-cells with environmental barriers due to their small number, the Wilcoxon rank sum test for nonparametric statistical comparison is used.

\section{Results and Findings}

As a result of data collection, 2,744 EDR samples were collected in the test site. Specifically, 41 samples were located on the grid-cells with the six environmental barriers and 2,703 samples were located on the other grid-cells without environmental barriers. Table 2 shows the descriptive statistics of the collective stress between two categories of gird-cells. The result of Wilcoxon rank sum test indicated that the collective stress metric (i.e., frequency of high-stress on a grid) on the grid-cells with environmental barriers (Median $=0.500$, Mode $=0.500)$ are significantly higher than on the grid-cells without environmental barriers (Median $=0.087$, Mode $=$ $0.000), z=3.2446, p<0.01$. This result demonstrates that the collective stress metric has indication of environmental barriers on the grid-cell. The finding of this study shows the potential of the proposed wearable-based collective sensing approach to distinguish the locations of environmental barriers by spatially aggregating multiple elderly individuals' EDA data.

Table 2 Descriptive Statistics: Collective Stress between Grid-cells with Barriers and without Barriers

\begin{tabular}{lll}
\hline Statistics & $\begin{array}{l}\text { Grid-cells } \\
\text { with Barriers }\end{array}$ & $\begin{array}{l}\text { Grid-cells } \\
\text { without Barriers }\end{array}$ \\
\hline Number & 6 & 225 \\
Mean (SD) & $0.499(0.277)$ & $0.167(0.240)$ \\
Median & 0.500 & 0.077 \\
Mode & 0.500 & 0.000 \\
\hline
\end{tabular}

In addition, the proposed approach could identify several timedependent barriers that the one-time site survey could not detect. Figure 3 is the spatial density estimation of the collective stress on the test site. Besides the six environmental barriers, Areas 7 and 8 display high values of the collective stress. Area 7 contains the driving way to which the main entrance of the senior apartment is connected. Also, in the area, there is a bus stop closest to the apartment. The authors found that high stress samples in Area 7 were collected when the elderly subjects interacted with automobiles such as crossing a driving road or waiting and getting on and off the buses. Area 8 is just an unoccupied ground. Therefore, snow on the area was not cleaned though the elderly subjects sometimes passed over the area. The high stress samples on the area might be caused by walking over the snowy ground. Since the current discontinuous survey-based attempts might not be able to detect such time-dependent barriers, this finding highlights the advantage of the proposed wearable-based collective sensing.

Although this study shows the feasibility of the proposed wearable-based collective sensing for detection of the elderly's environmental barriers, there are several limitations. First, the number of stress samples located on the test site was small mainly due to limited number of subjects and period of the data collection. If the authors gather more data from more subjects for longer period, it would be possible to conduct more indepth analysis for understanding characteristics of environmental barrier. For example, by comparing collective stress between different barriers, on which barriers people feel more stress can be understood. Also, this study did not consider the impact of the elderly's diverse characteristics such as their physical functionality, gender, or assistive device use. Since such characteristics can affect the elderly's interactions with environmental barriers, future research should incorporate the elderly's characteristics and investigate their impact on stress and the performance of the proposed approach.

Figure 3 Collective Stress Distribution, (1)-(6) Identified Barriers, (7)-(8) Areas of Time-Dependent Barriers

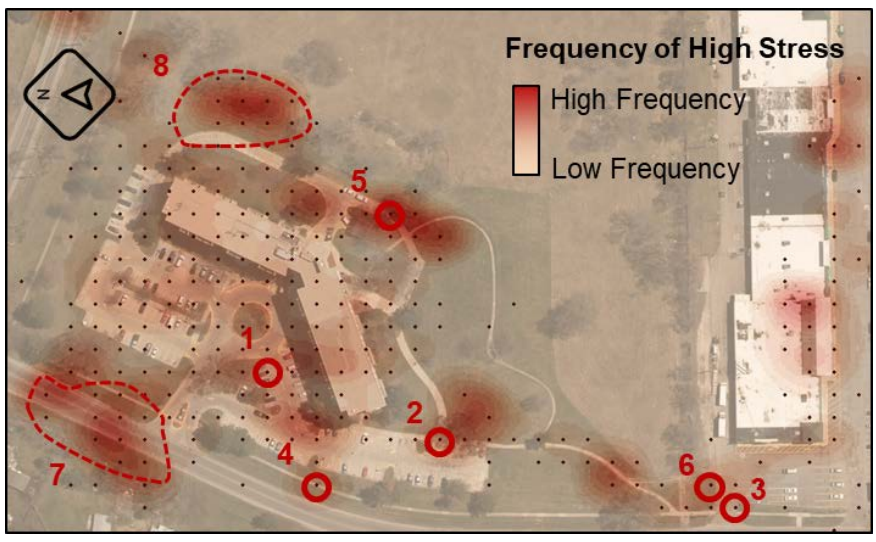

\section{Conclusion}

This study proposes a wearable-based collective sensing approach to detect the elderly's environmental barriers for advancing their mobility. To test the proposed approach, collective stress on locations of the test site were measured based on the data collected from 10 elderly subjects' daily trips for two weeks. Then, the measured collective stress was statistically compared between locations with environmental barriers and without environmental barriers. As a result, the collective stress on locations with environmental barriers are statistically higher, which implies that the high collective stress is well associated with the existence of environmental barriers on the location. This finding shows the feasibility of the proposed wearable-based collective sensing approach for detection of the elderly's environmental barriers. The finding also can contribute to the body of knowledge by providing the direction to understand locational stress-inducing factors by spatially aggregating multiple people's physiological data.

\section{Acknowledgements}

This study was supported by the Exercise and Sport Science Initiative (ESSI-2018-4), the Urban Collaboratory in the University of Michigan, and the National Science Foundation - United States (\# 1800310). Also, the authors wish to acknowledge Brenda Stumbo, Ypsilanti Township Supervisor, 
and Denise M. McKalpain, Service Coordinator at Clark East Tower for their help in data collection.

\section{References}

Behrens, CN, et al. (2004) Bayesian analysis of extreme events with threshold estimation. Statistical Modelling, 4(3), 227-244.

Bendall, M, et al. (1989) Factors affecting walking speed of elderly people. Age and ageing, 18(5), 327-332.

Bond, LJ (1999) Predictive engineering for aging infrastructure. Proc., Nondestructive Evaluation of Utilities and Pipelines III, International Society for Optics and Photonics, 2-14.

Boucsein, W (2012) Electrodermal activity, Springer Science $\&$ Business Media.

Bornoiu, IV, and Grigore, O (2013) A study about feature extraction for stress detection. Proc., Advanced Topics in Electrical Engineering (ATEE), 2013 8th International Symposium on, IEEE, 1-4.

Brighetti, G, et al. (2014) Do emotions affect insurance demand? Review of Behavioral Finance, 6(2), 136-154.

Carreau, J, and Bengio, Y (2009) A hybrid Pareto model for asymmetric fat-tailed data: the univariate case. Extremes, 12(1), 53-76.

Chang, C-Y, et al. (2013) Physiological emotion analysis using support vector regression. Neurocomputing, 122, 79-87.

Choi, J, et al. (2012) Development and evaluation of an ambulatory stress monitor based on wearable sensors. IEEE transactions on information technology in biomedicine, 16(2), 279-286.

Cacioppo, JT, et al. (2007) Handbook of psychophysiology, Cambridge University Press.

Centers for Disease Control and Prevention (2018) U.S. physical activity statistics. http://apps.nccd.cdc.gov/PASurveil lance/DemoCompareResultV.asp? Year $=2007 \&$ State $=0 \&$ Cat $=$ $1 \& \mathrm{CI}=\#$ resultS. (accessed 25/12/2018).

Decker, BL (1986) World geodetic system 1984. Defense Mapping Agency Aerospace Center St Louis Afs Mo.

Drachen, A, et al. (2010) Correlation between heart rate, electrodermal activity and player experience in first-person shooter games. Proc., Proceedings of the 5th ACM SIGGRAPH Symposium on Video Games, ACM, 49-54.

Federal Highway Administration (1995) Our Nation's Travel: 1995 NPTS Early Results Report. US Dept of Transportation; Washington, DC.

Frigessi, A, et al. (2002) A dynamic mixture model for unsupervised tail estimation without threshold selection. Extremes, 5(3), 219-235.

Greco, A, et al. (2016) cvxEDA: A convex optimization approach to electrodermal activity processing. IEEE Transactions on Biomedical Engineering, 63(4), 797-804.

Guralnik, JM, et al. (1993) Maintaining mobility in late life. I. Demographic characteristics and chronic conditions. American Journal of Epidemiology, 137(8), 845-857.
Heikenfeld, J, et al. (2018) Wearable sensors: modalities, challenges, and prospects. Lab on a Chip, 18(2), 217-248.

Iwaksson, S, and Isacsson, А (1996) Housing Standards, Environmental Barriers in the Home and Subjective General Apprehension of Housing Situation among the Rural Elderly. Scandinavian Journal of Occupational Therapy, 3(2), 52-61.

Jebelli, H, et al. (2018) Feasibility study of a wristband-type wearable sensor to understand construction workers' physical and mental status. Proc., Construction Research Congress.

Kim, H, et al. (2016) A people-centric sensing approach to detecting sidewalk defects. Advanced Engineering Informatics, 30(4), 660-671.

King, AC, and Guralnik, JM (2010) Maximizing the potential of an aging population. JAMA, 304(17), 1944-1945.

Lawton, MP, and Nahemow, L (1973) Ecology and the aging process.

Lockett, D, et al. (2005) Through seniors' eyes: an exploratory qualitative study to identify environmental barriers to and facilitators of walking. CJNR (Canadian Journal of Nursing Research), 37(3), 48-65.

MacDonald, A, et al. (2011) A flexible extreme value mixture model. Computational Statistics \& Data Analysis, 55(6), 2137 2157.

Manton, KG (1988) A longitudinal study of functional change and mortality in the United States. Journal of Gerontology, 43(5), S153-S161.

Mattson, JW (2011) Aging and mobility in rural and small urban areas: A survey of North Dakota. Journal of Applied Gerontology, 30(6), 700-718.

Metz, DH (2000) Mobility of older people and their quality of life. Transport policy, 7(2), 149-152.

Machigan Department of Natural Resources (2005) Map Projections.

https://www.michigan.gov/documents/DNR_Map_Proj_and_ MI_Georef_Info_20889_7.pdf (Accessed 25/12/2018).

Mollenkopf, H, et al. (1997) Outdoor mobility and social relationships of elderly people. Archives of gerontology and geriatrics, 24(3), 295-310.

Pappens, M, et al. (2011) Psychophysiological responses to inspiratory resistive loads. International journal of psychophysiology, 80(2), 161-165.

Preedy, VR, and Watson, RRR (2010) Handbook of disease burdens and quality of life measures. Handbook of disease burdens and quality of life measures.

Rantakokko, M, et al. (2009) Fear of moving outdoors and development of outdoor walking difficulty in older people. Journal of the American Geriatrics Society, 57(4), 634-640.

Rockwood, K, et al. (2004) Prevalence, attributes, and outcomes of fitness and frailty in community-dwelling older adults: report from the Canadian study of health and aging. The Journals of Gerontology Series A: Biological Sciences and Medical Sciences, 59(12), 1310-1317.

Rosenberg, DE, et al. (2012) Outdoor built environment barriers and facilitators to activity among idlife and older 
adults with mobility disabilities. The Gerontologist, 53(2), 268-279.

Setz, C, et al. (2010) Discriminating stress from cognitive load using a wearable EDA device. IEEE Transactions on information technology in biomedicine, 14(2), 410-417.

Silverman, BW (2018) Density estimation for statistics and data analysis, Routledge.

Singh, RR, et al. (2014) Assessment of driver stress from physiological signals collected under real-time semi-urban driving scenarios. International Journal of Computational Intelligence Systems, 7(5), 909-923.

Sweeney, KT, et al. (2012) Artifact removal in physiological signals-Practices and possibilities. IEEE transactions on information technology in biomedicine, 16(3), 488-500.

Ursin, H, and Eriksen, HR (2004) The cognitive activation theory of stress. Psychoneuroendocrinology, 29(5), 567-592.

Walker, A (2002) A strategy for active ageing. International social security review, 55(1), 121-139.

Wijsman, J, et al. (2011) Towards mental stress detection using wearable physiological sensors. Proc., Engineering in Medicine and Biology Society, EMBC, 2011 Annual International Conference of the IEEE, IEEE, 1798-1801.

World Health Organization (2007) Global age-friendly cities: A guide, World Health Organization.

Yang, K, et al. (2017). Collective sensing of workers' gait patterns to identify fall hazards in construction. Automation in Construction, 82, 166 